\title{
Functional Super Read Out Driver Demonstrator for the Phase II Upgrade of the Atlas Tile Calorimeter
}

\section{Fernando Carrió Argos}

Department of Electronic Engineering, University of

Valencia

IFIC (CSIC-UV)

On behalf of the ATLAS Tile Calorimeter System 


\section{Outline}

- Introduction

- Phase II Upgrade

- Optical Link Card development (OLC)

- Optical Multiplexer Board (OMB)

- sROD demonstrator tests

- sROD demo board

- Conclusions 


\section{Introduction}

- ATLAS Hadronic Tile Calorimeter at LHC (CERN)

- Materials:

- Iron as passive material

- Plastic scintillating plates

- Strucuture:

- One central barrel

- Two extended barrels
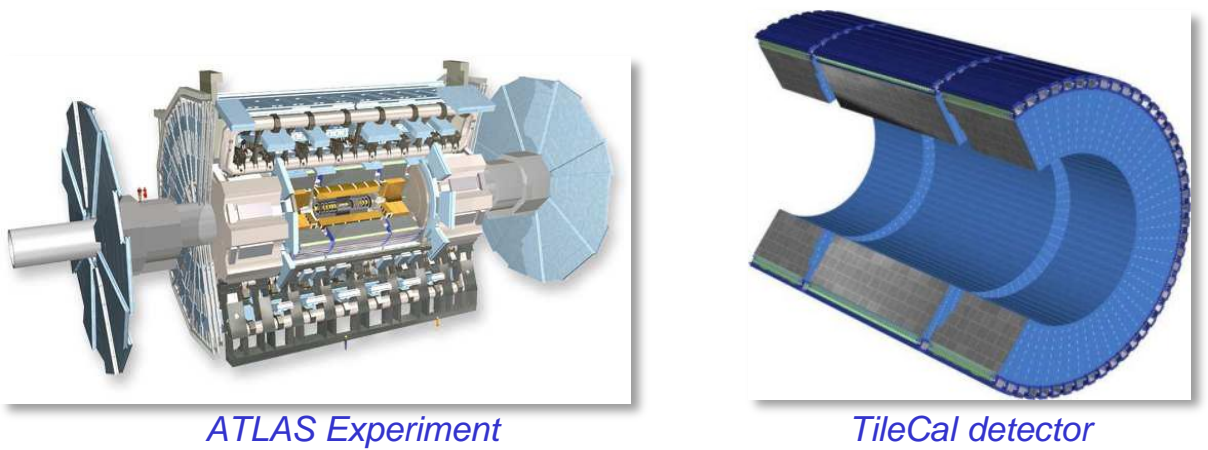

- Energy deposited in cells of barrels generates light

- Front-end electronics digitizes signals @ 25ns

- Located at the outermost section of the Calorimeter

- Send digital samples with optical fibers to the backends electronics

- Back-end electronics receives digitized information

- Main component is the ReadOut Driver (ROD)

» $9 U \mathrm{VME}$ format

»SFP optical connectors

» 2 PU equipped with TMS320C64 DSPs

»Data format

" Management

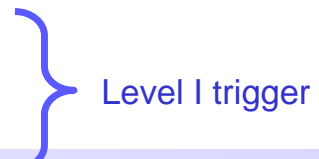

"Transmission

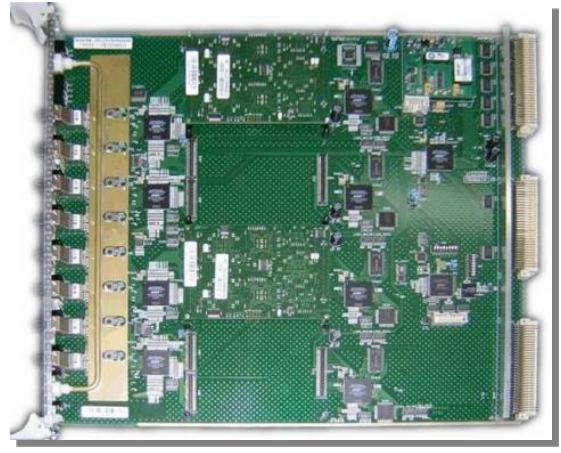

Read Out Driver board 


\section{Phase II Upgrade}

- LHC increases luminosity in a factor of 10

- Increase of data rate between front-end and backend electronics

- Digital trigger to be implemented

- Higher resolution needed

- Radiation tolerance must be increased

- Highly reliable data transmission protocols must be deployed

- GigaBit Transceiver protocol

- Redesign of the front-end and back-end electronics

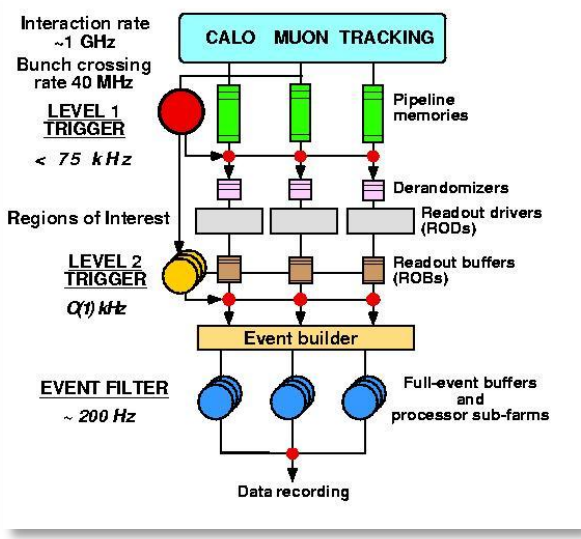

Atlas trigger system

- New proposed back-end electronics implies

- Increased data bandwidth

- SNAP12 optical connectors

- FPGA with embbebed SerDes

- Increase in processing requirements

- High performance FPGA with DSP blocks

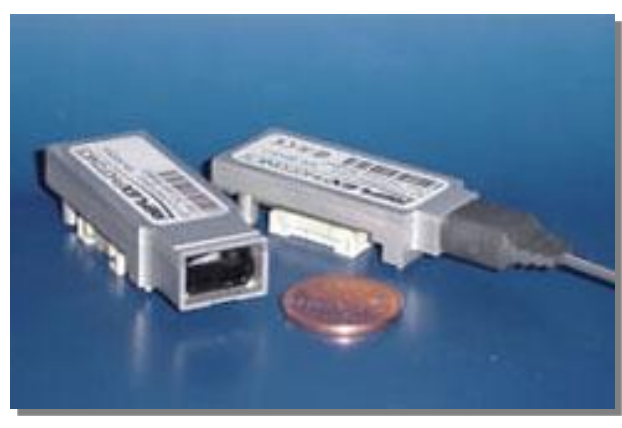

SNAP12 optical connectors 


\section{Optical Link Card}

- Description

- Specifications

- ROD double PU Board format (4.72 in x 6.85 in)

- 12 input channels up to 75 Gbps

- 12 output channels up to 75 Gbps

- Technical details

- Altera Stratix II GX EP2SGX60E FPGA

- 1152 pins

_ 12 high speed transceivers @ 6.375Gbps

- $534 \mathrm{l} / \mathrm{Os}$

- 2 SNAP12 connectors (12 fibers @ 6.25Gbps)

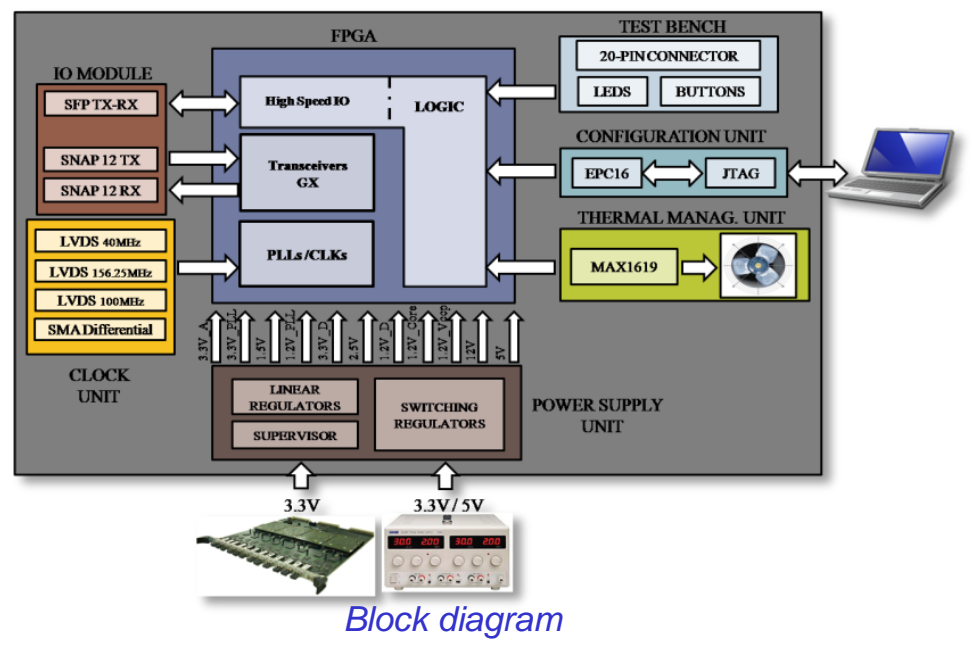

- 1 SFP connector (1 input @ 1Gbps, 1 output @ 1Gbps)

- 12 copper layers

- 6 layers - 0.6 mils thick for signals

- 6 layers - 1.2 mils thick for planes

- Different thickness of dielectric: 2.5, 4 and 6 mils

- Need high capacitance between planes but sufficient thickness for striplines

- Over 2000 routes: 5 mils width

- Over 2400 vias: 10 mils

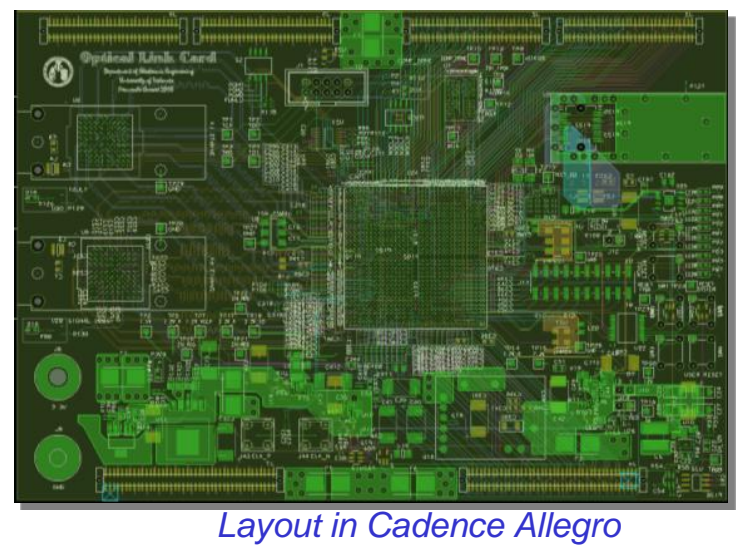




\section{Optical Link Card}

- Tests developed

- Test 1: Measurement of latency and maximum data bandwidth

- Raw data: 32 bit counter

- No protocol

- $\quad$ 6.25 Gbps per fiber

- Latency of 108ns - 17 clock cycles

- Total measured bandwidth of 75 Gbps

- Power consumption 11.71 W

- Test 2: 1 link GBT

- 1 link GBT protocol

- Receiver and transmitter located in different GX transceiver block

- 48 hours without errors

- BER of $3.61 \cdot 10^{-15}$ for a confidence of $95 \%$

- Total bandwidth of $4.8 \mathrm{Gbps}$

- Power consumption 7.128 W

- Test 3: 12 link GBT

- 12 link GBT protocol

- Receiver and transmitter located in different GX transceiver block

- Internal FIFO compensation needed

- BER of $6.05 \cdot 10^{-16}$ for a confidence of $95 \%$

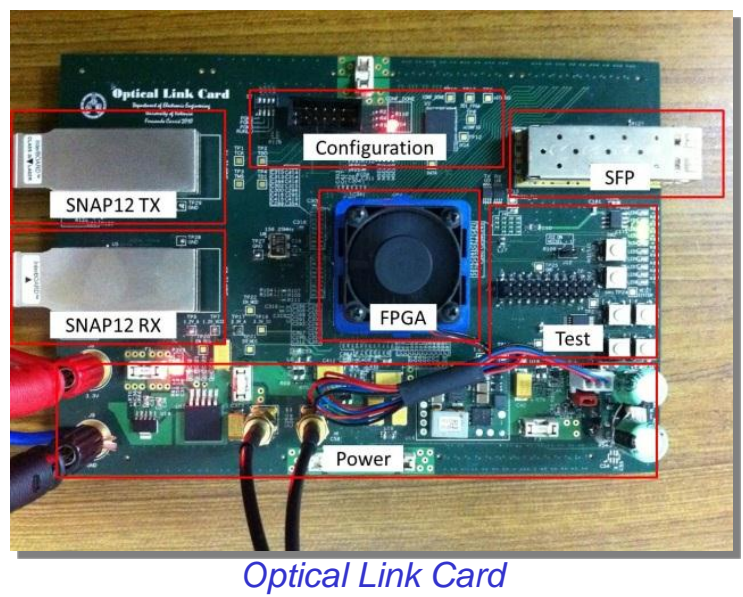

- Total bandwidth of $57.6 \mathrm{Gbps}$

- Power consumption 14.025 W

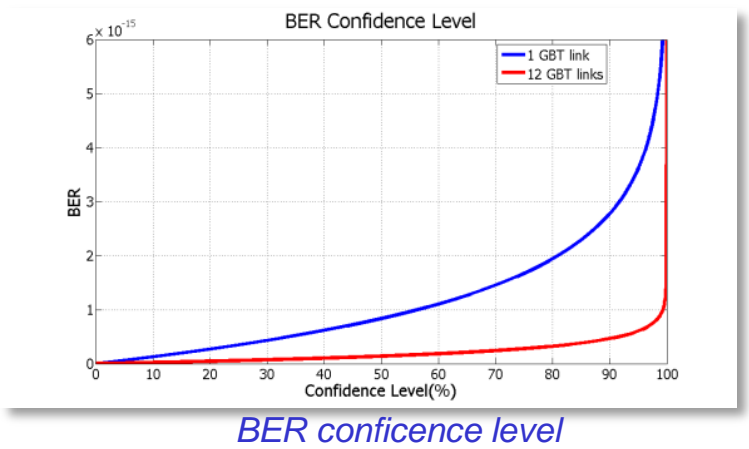




\section{Optical Multiplexer Board}

- Add redundancy to data transmission

- Two optical fibers

- Same data from front-end electronics

- Specifications

- $\quad$ gU format board

- 16 input channels up to 10.24 Gbps

- 8 output channels up to $5.12 \mathrm{Gbps}$

- Technical details

- 8 Altera Cyclone I EP1C12 FPGA

- $\quad$ CRC checking

- Link control

- 1 Altera ACEX EP1K100

- VME interface

- 1 TTCrx chip

- 12 SFP optical connectors

- 8 TX channels @640Mbps (HDMP-1032)

- 16 RX channels@640Mbps (HDMP-1034)

- 10 copper layers

- More than 1200 components

- Tests

- 2100 hours

- BER better than $10^{-13}$ for a confidence level of $95 \%$

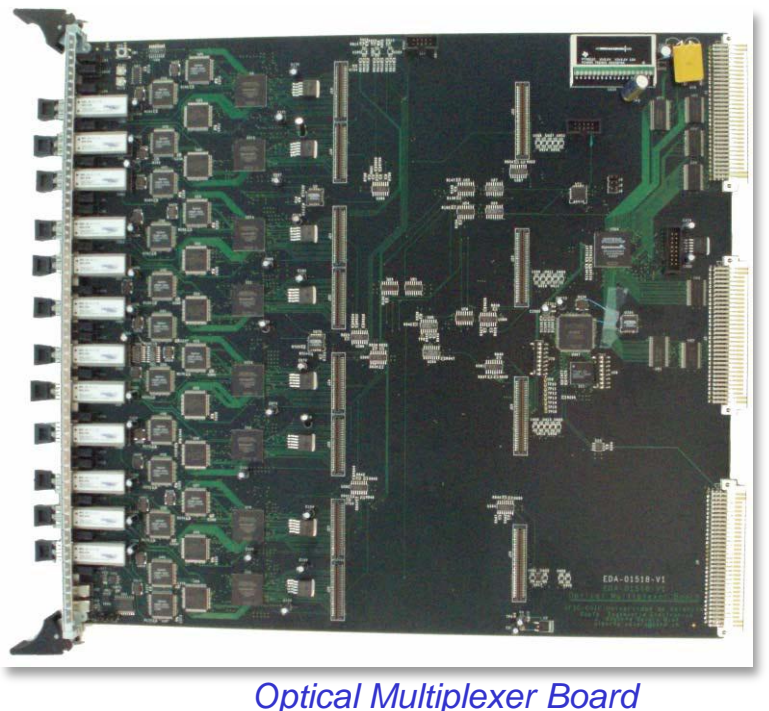

Optical Multiplexer Board

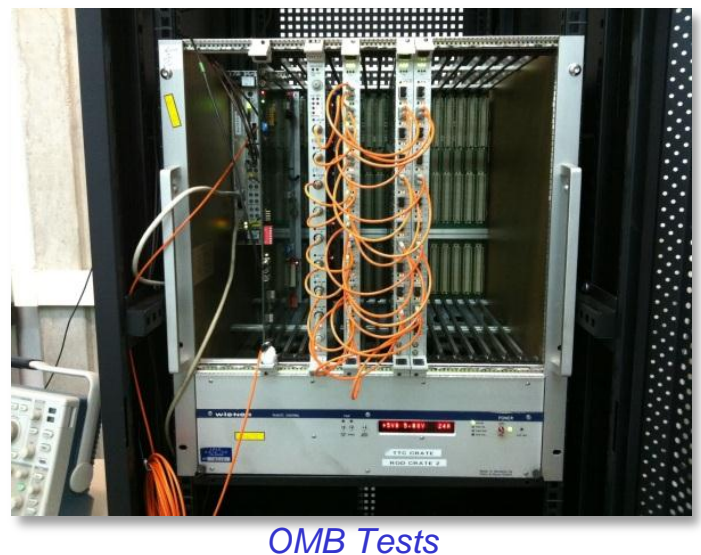




\section{sROD demonstrator tests}

- Functional sROD Demonstrator

- OLC plugged on an OMB

- Optical Link Card

- Function

- Send event data with GBT protocol @4.8Gbps

"Emulating Front-End electronics

- Receive sent data and decode it @4.8Gbps

"Emulating Back-End electronics

- Used devices

- Altera Stratix II GX FPGA

- SNAP12 optical connectors

»1 TX channel @ 4.8Gbps

》1 RX channel @ 4.8Gbps

- Optical Multiplexer Board

- Function

- Receive event data through PU connectors

- Send event data to ROD @640Mbps

- Used devices

- Altera Cyclone I FPGA

- SFP optical connector

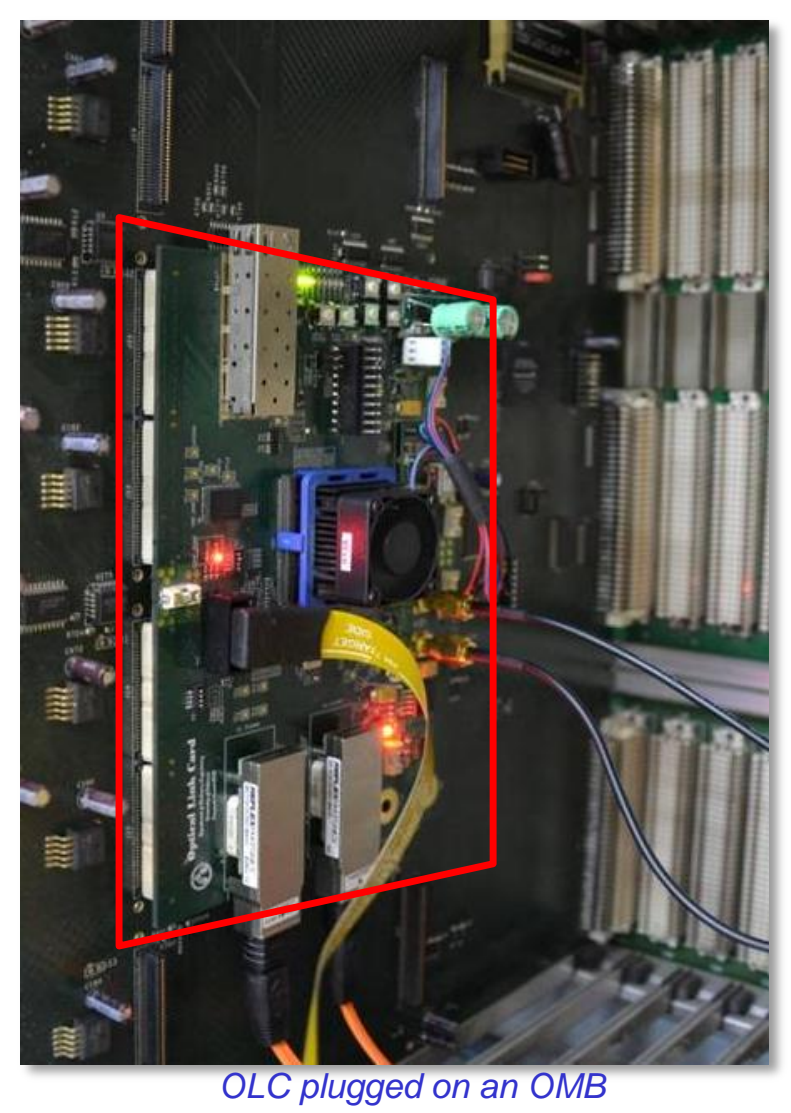

»1 TX@640Mbps 


\section{sROD demonstrator tests}

- Block diagram

- Front-end emulation

- Event data from ROM (OLC)

- Stored in Stratix II GX

-1 TX GBT link @4.8Gbps (OLC)

- TX SNAP12 optical connector
- Back-end emulation

-1 RX GBT link @4.8Gbps (OLC)

- RX SNAP12 optical connector

- $\mathrm{CRC}$ data check (OMB)

- 1 G-link @640Mbps (OMB)

- Send event data to ROD

- 1 TX SFP optical connector
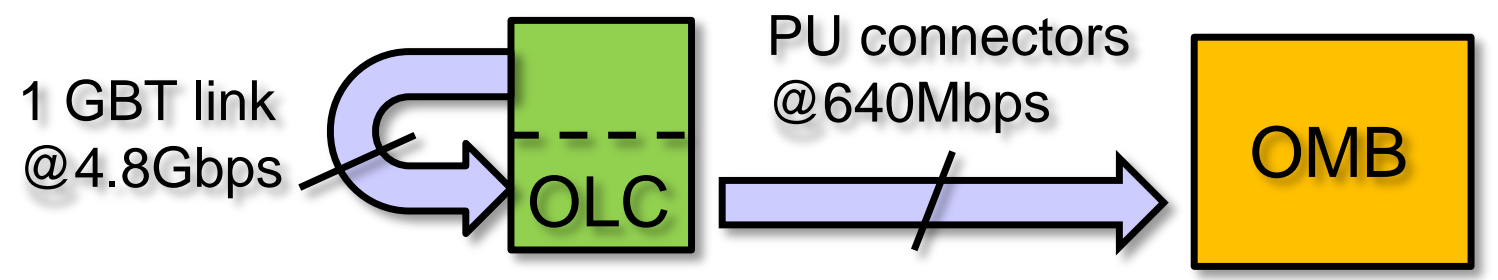

G-link
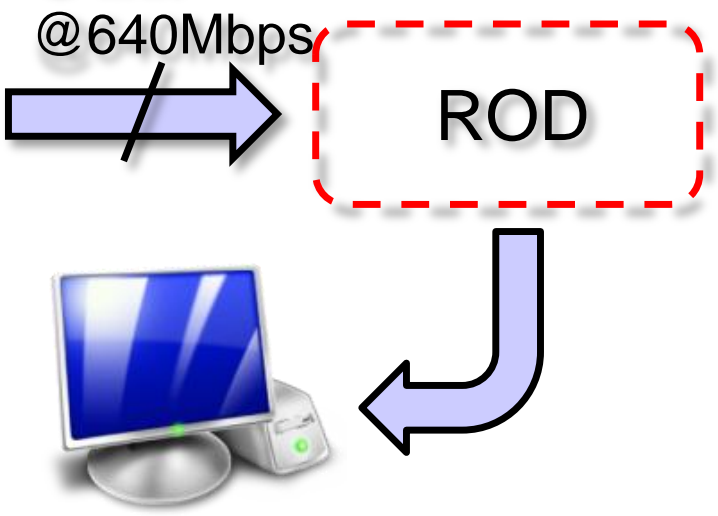


\section{VNIVERSITAT ID VALẼNCIA}

\section{sROD demonstrator tests}

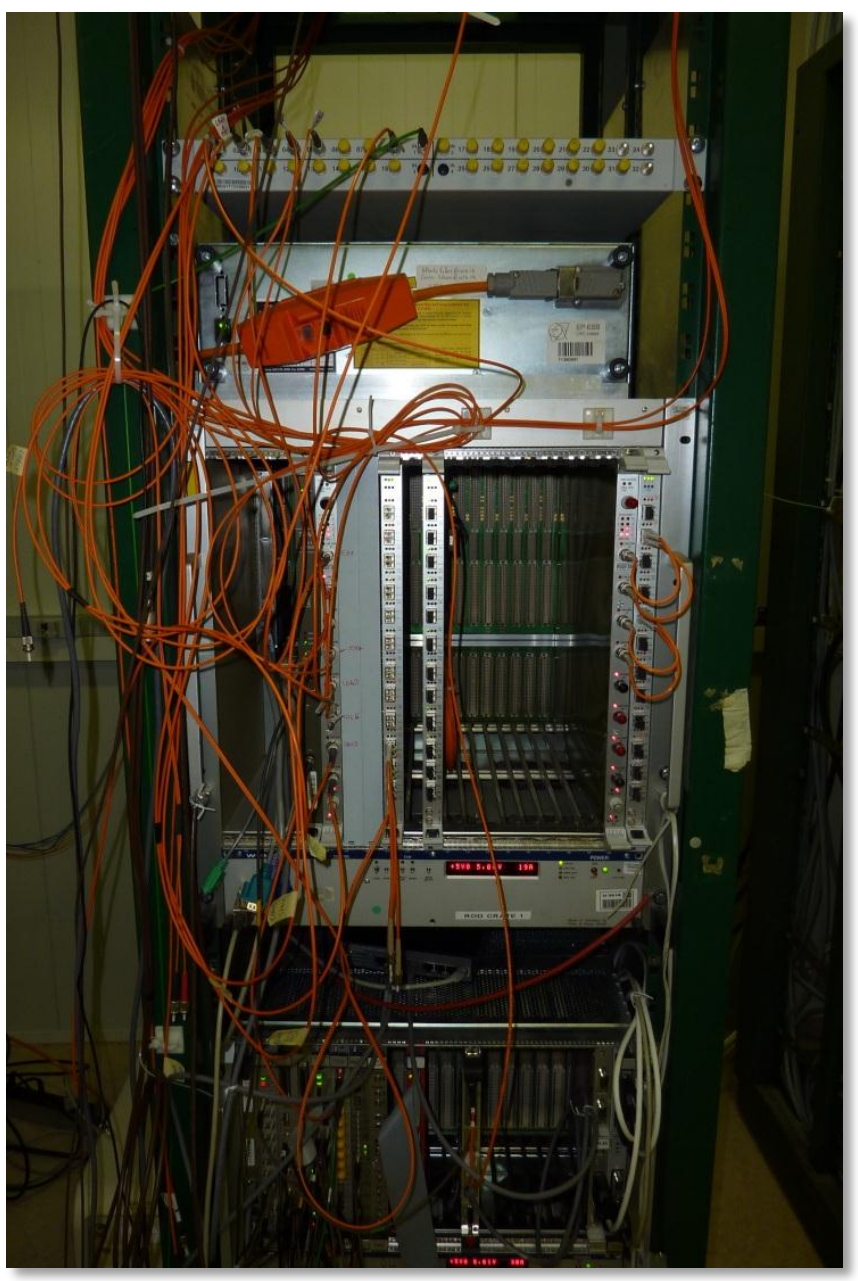

sROD demonstrator testbench 


\section{sROD demo board}

- Next step for the Upgrade phase II

- Aim of the sROD demo board

- Read-Out of a complete super-drawer (Upgrade architecture)

- Transmit trigger data to the L1Calo

- Send data at L1 trigger rate to the ROS

- Physical features:

- ATCA format

- Ethernet interface

- Remote configuration

- Remote process monitoring

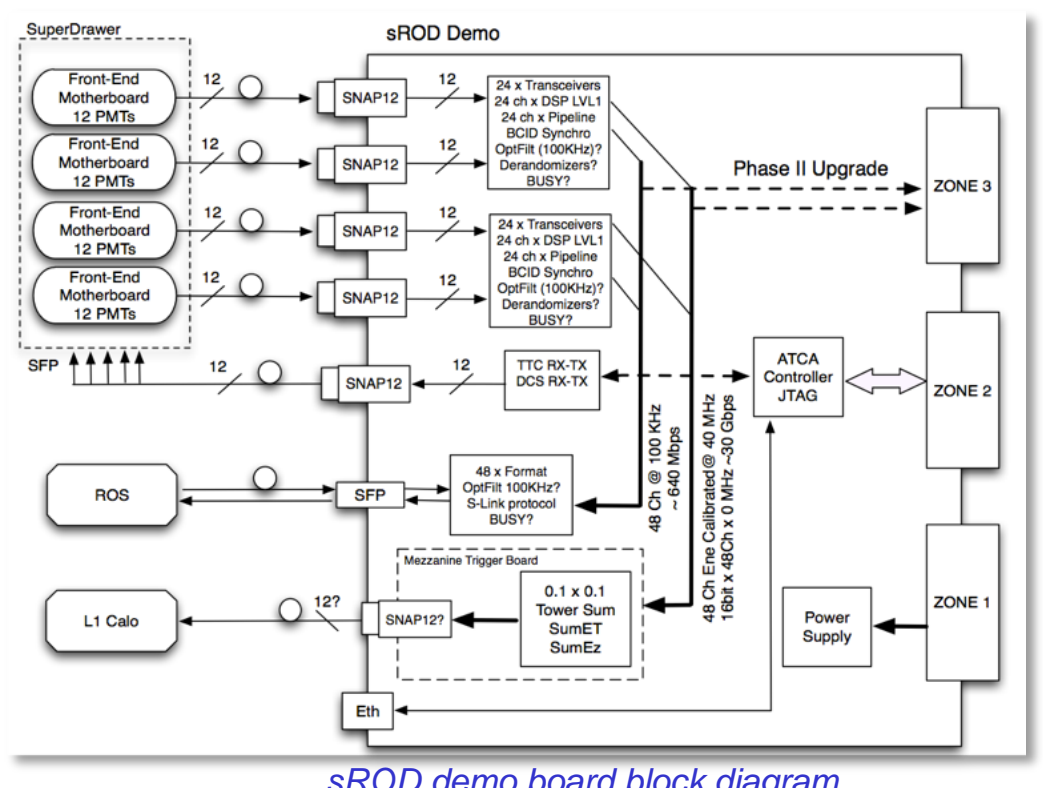




\section{Conclusions}

- Developed an evaluation board for test SNAP12 connectors

- Optical Link Card

- A super Read Out Driver demonstrator has been presented

- OLC plugged on an OMB

- Transmission data rate @ 4.8Gbps

- Reception data rate @ 4.8Gbps

- Basis for a new project: sROD demo board

- Read-out a whole module (super-drawer)

- 4 RX SNAP12

- 2 TX SNAP12 


\section{VNIVERSITAT \\ ID VALĖNCIA}

\section{Thank you}

